\title{
Electron backscattering simulation in Geant4
}

\author{
Paolo Dondero ${ }^{\mathrm{a}, *}$, Alfonso Mantero ${ }^{\mathrm{a}}$, Vladimir Ivanchencko ${ }^{\mathrm{b}, \mathrm{c}, \mathrm{g}}$, Simone Lotti ${ }^{\mathrm{d}}$, Teresa Mineo ${ }^{\mathrm{e}}$, \\ Valentina Fioretti ${ }^{\mathrm{f}}$ \\ a SWHARD S.R.L., Via Greto di Cornigliano 6r, 16152 Genova, Italy \\ ${ }^{\mathrm{b}}$ CERN, Geneve 23 1211, Switzerland \\ c Geant4 Associate International Ltd, 9 Royd Terrace, Hebden Bridge HX7 7BT, United Kingdom \\ d INAF/IAPS Roma, Via del Fosso del Cavaliere, 100, 00133 Roma, Italy \\ e INAF/IASF Palermo, Via Ugo La Malfa 153, 90146 Palermo, Italy \\ ${ }^{\mathrm{f}}$ INAF/IASF Bologna, Via P. Gobelli 101, 40129 Bologna, Italy \\ ${ }^{\mathrm{g}}$ Tomsk State University, Leninsky Pr. 36, 634050 Tomsk, Russia
}

A R T I C L E I N F O

\section{Keywords:}

Geant4

Validation

Backscattering

Electrons

\begin{abstract}
A B S T R A C T
The backscattering of electrons is a key phenomenon in several physics applications which range from medical therapy to space including AREMBES, the new ESA simulation framework for radiation background effects. The importance of properly reproducing this complex interaction has grown considerably in the last years and the Geant4 Monte Carlo simulation toolkit, recently upgraded to the version 10.3, is able to comply with the AREMBES requirements in a wide energy range. In this study a validation of the electron Geant 4 backscattering models is performed with respect to several experimental data. In addition a selection of the most recent validation results on the electron scattering processes is also presented. Results of our analysis show a good agreement between simulations and data from several experiments, confirming the Geant 4 electron backscattering models to be robust and reliable up to a few tens of electronvolts.
\end{abstract}

\section{Introduction}

Scattering of particles is a critical component of every Monte Carlo transport code, being one of the fundamental processes to represent the particle evolution within matter. The ESA AREMBES (ATHENA Radiation Environment Models and X-ray Background Effects Simulators) consortium aims to develop a new space radiation background effects simulator for the ATHENA [1] X-ray telescope. The AREMBES simulator is based on Geant4 [2-4], a Monte Carlo trackstructure toolkit designed for the simulation of particle transport through matter. Geant 4 addresses the scattering process with different approaches, accuracy, and CPU usage [5]. The version 10.3 of Geant4, released in December 2016, includes several improvements as well as new developments in hadronic and electromagnetic physics interactions.

Within the AREMBES framework the simulation of low-energy electron scattering is fundamental. Secondary electrons are produced by proton scattering on the materials surrounding the X-ray instruments. These particles can be completely absorbed by the detectors or can backscatter on their surfaces, avoiding de facto any means of detection by anti-coincidence techniques and leading to a dominant contribution to the overall background of both ATHENA focal plane detectors $[6,7]$. Given the relevance of the subject a detailed study, reported in this paper, was carried on by a synergy of AREMBES and Geant4 Collaboration members in order to evaluate the real capability of Geant 4 to accurately reproduce the electron backscattering process.

In the first part of this paper an overview of electron scattering models and Geant4 internal validation will be presented. In the second part the electron backscattering process will be addressed by comparing the simulation with respect to a collection of experimental datasets available in literature for a variety of materials and energy regimes.

\section{Electron scattering in Geant4}

\subsection{Scattering models}

The Geant4 toolkit offers several scattering models applicable to different particles in a wide energy range. In the following paragraphs those models are briefly discussed, focusing on their applicability to electrons.

\footnotetext{
* Corresponding author.

E-mail address: paolo.dondero@cern.ch (P. Dondero).
} 


\subsubsection{Urban multiple scattering model}

The multiple scattering model (MSC in the following) developed by Laszlo Urban [8] based on the Lewis theory [9] has been the Geant4 default model of multiple scattering for a long time. The function describing the scattering distribution shape has been tuned on the basis of various electron scattering datasets. The Urban model is applicable to any particle, but in recent Geant 4 versions it is used only for electrons, positrons, and ions. In particular for electrons and positrons this model is exploited for energies below $100 \mathrm{MeV}$ since the parametrisations used by the model are optimized for this energy range. The only exception is represented by the emstandardopt 3 (opt3 in the following) electromagnetic physics configuration, where the Urban model is used for all particles and all energies.

\subsubsection{Combined multiple and single scattering model}

In the recent Geant 4 versions including 10.3 a model that combines multiple and single scattering, called Wentzel-VI, is provided $[4,5]$. This combined model can be used with low CPU usage for all charged particles and all energies, except electron and positron below $100 \mathrm{MeV}$.

\subsubsection{Goudsmit-Saunderson multiple scattering model}

This model is based on Goudsmit-Saunderson theory [10,5] and use some algorithms of the EGSnrc multiple scattering model developed by Kawrakow and Bielajew [11]. In this model at each step a sampling of
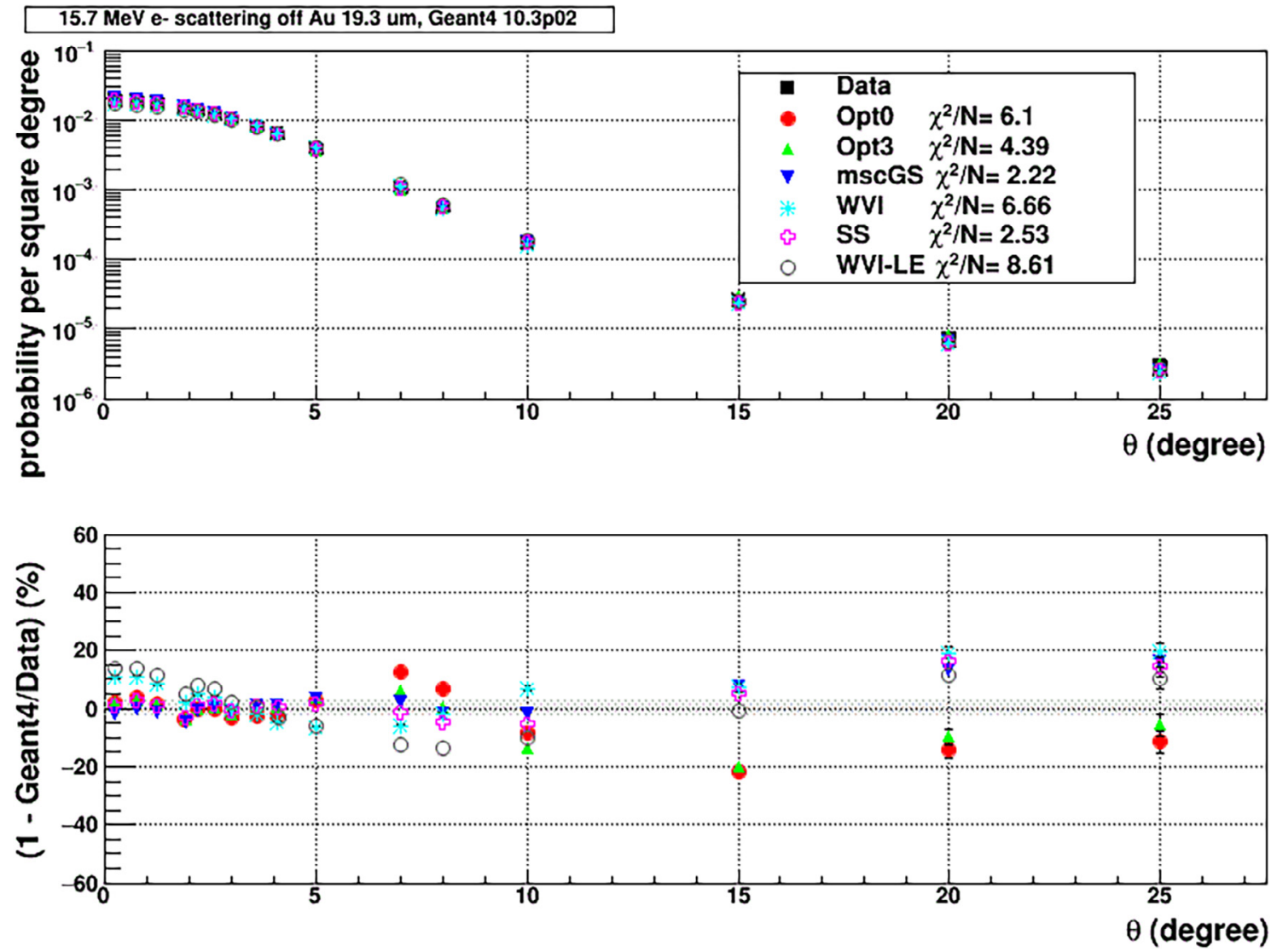

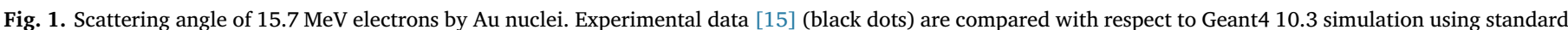

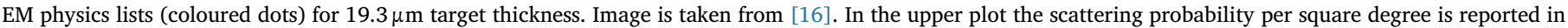
function of the scattering angle $\theta$. In the lower plot the deviation between data and simulation in percentage is reported in function of the scattering angle $\theta$.
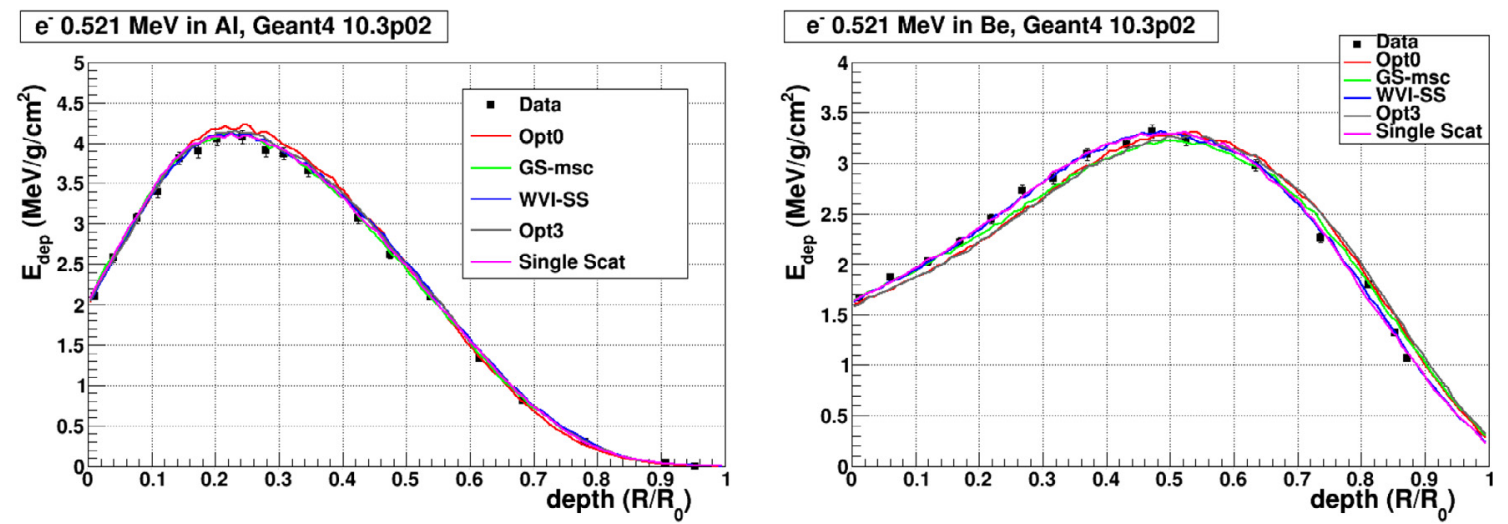

Fig. 2. Dose deposition of $0.5 \mathrm{MeV}$ electron beam in $\mathrm{Al}$ (left plot) and Be (right plot) as a function of depth in the unit of electron range. Black points are experimental data from [17]. Colored curves are the simulation results obtained with Geant4 10.3 using various standard electromagnetic physics lists. Image is taken from [5]. 
Table 1

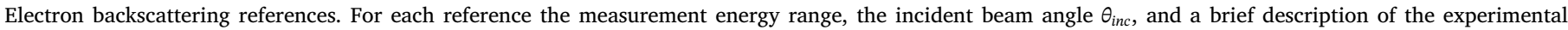
apparatus is reported.

Electron backscattering experimental datasets

\begin{tabular}{|c|c|c|c|}
\hline Reference & Energy range & $\theta_{i n c}\left({ }^{\circ}\right)$ & Experimental method \\
\hline Lockwood et al. $[22,23]$ & $0.1 \mathrm{MeV}-1 \mathrm{MeV}$ & $0,15,30,45,60,75$ & $\begin{array}{l}\text { Calorimetric measure (indirect). Possible systematics at low energies discussed in the } \\
\text { paper }\end{array}$ \\
\hline L. Reimer and C. Tollkamp [25] & $1 \mathrm{keV}-30 \mathrm{keV}$ & 0 & Polarized grid $( \pm 50 \mathrm{~V})$ \\
\hline Bongeler et al. [26] & $1 \mathrm{keV}-10 \mathrm{keV}$ & 0 & Polarized grid $( \pm 50 \mathrm{~V})$ \\
\hline R. Shimizu $[29,30]$ & $1 \mathrm{keV}-15 \mathrm{keV}$ & 0 & Polarized grid. Three grids used with different biases, collector at $90 \mathrm{~V}$ \\
\hline H.E. Bishop [27] & $5 \mathrm{keV}-30 \mathrm{keV}$ & 0 & Double polarized target \\
\hline E. Weinryb, J. Philibert [31] & $5 \mathrm{keV}-30 \mathrm{keV}$ & 0 & Polarized grid $(-160 \mathrm{~V})$ \\
\hline K.F.J. Heinrich $[28]$ & $30 \mathrm{keV}$ & 0 & Two region target \\
\hline G. Neubert, S. Rogaschewski [32] & $15 \mathrm{keV}-60 \mathrm{keV}$ & $\begin{array}{l}0,10,20,30,40,50,60 \\
70,75,80\end{array}$ & Double polarized target \\
\hline H. Kanter $[33,34]$ & $2 \mathrm{keV}-20 \mathrm{keV}$ & 0 & Polarized grid $-100 \mathrm{~V}$, collector at $-50 \mathrm{~V}$. Half target bulk, half thin film \\
\hline H. Drescher, L. Reimer, H. Seidel [35] & $10 \mathrm{keV}-100 \mathrm{keV}$ & 0 & Single polarized target \\
\hline D.B. Wittry [36] & $5 \mathrm{keV}, 30 \mathrm{keV}$ & 0 & Two region target, composite materials \\
\hline I.M. Bronstein, B.S. Fraiman [37] & $0.1 \mathrm{keV}-4 \mathrm{keV}$ & 0 & \\
\hline M.M. El Gomati, A.M.D. Assad [19] & $0.6 \mathrm{keV}-6 \mathrm{keV}$ & 0 & Polarized grid $( \pm 50 \mathrm{~V})$, surface cleaning \\
\hline
\end{tabular}

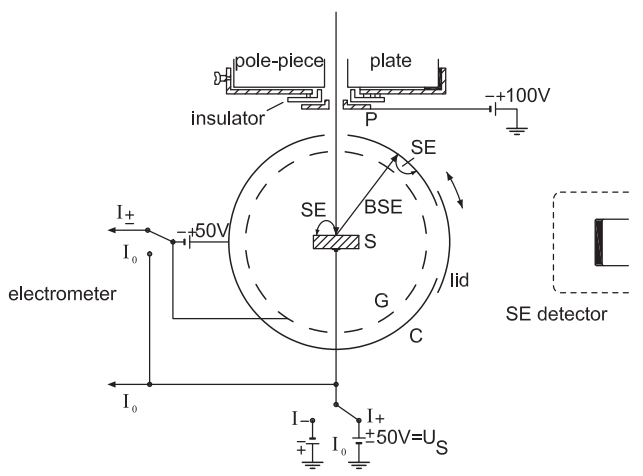

single, multiple or no scattering is performed for electrons and positrons depending on the number of interactions along the path. A physics list called emstandardGS (GS in the following, see [12]) includes the Goudsmit-Saunderson model for multiple scattering of electrons and positrons below $100 \mathrm{MeV}$.

\subsubsection{Single scattering models}

Also single scattering model is available within Geant4. It is applicable to all particle types and all energies. It is available within a dedicated standard EM physics list called emstandardSS (SS in the following). An alternative implementation is provided for electrons and positrons [13], in which the Mott correction to the scattering formula is included.

\subsection{Validation}

Electron scattering is regularly tested in various energy regimes by the Geant4 Collaboration. A forward scattering benchmark study is performed against 13 and $20 \mathrm{MeV}$ electrons data taken from [14]. Another comparison is performed with respect to the experimental data from [15], addressing scattering of $15.7 \mathrm{MeV}$ electrons by nuclei in thin gold foils. In Fig. 1 the experimental data (black squares) and the simulation results obtained with several standard EM physics lists are shown as a function of the scattering angle distribution. The best agreement (within several percent) is obtained with opt3 for small scattering angles. SS and Wentzel-VI provide a better agreement for angles above $10^{\circ}$, where the discrepancies do not exceed $20 \%$ for both the considered target thickness. Also the simulation of energy deposition in matter is an important general confirmation of the goodness of the scattering models used, and thus needs to be exhaustively validated. In
Fig. 3. Left plot: device used in [25] for the direct measurement of the electron backscattering coefficient and secondary electron yield. $S$ is the specimen, $\mathrm{G}$ is a $-50 \mathrm{~V}$ polarized grid, $\mathrm{C}$ is the collector, and $\mathrm{P}$ is a negatively biased plate to delay the secondary electrons coming down the column (polarized grid). Right plot: device used in [27]. The double target method is used. When the incident electron beam scatters on the first target $\mathrm{B} 1$, the second target $\mathrm{B} 2$ is used to measure the background current from the chamber walls (polarized double target).
Fig. 2 the standard EM physics lists are compared with Sandia Laboratories data on dose deposition of $0.5 \mathrm{MeV}$ electrons in light targets (Be and $\mathrm{Al}$ ). Total energy deposition in this test is directly dependent on the backscattering electron energy. Wentzel-VI and single scattering physics lists are the most accurate for the Be target. In the $\mathrm{Al}$ case all physics lists are in acceptable agreement with respect to experimental data.

\section{Electron backscattering simulations}

In this section a detailed investigation on the electron backscattering probability, nominally the case where the electrons are scattered in the backward direction by the interaction with the target surface, is presented. This phenomenon is a key variable to properly examine the electron transport and interaction with shielding for space instruments.

\subsection{Experimental setups}

Several experimental measurements of the electron backscattering coefficient (indicated as $\eta$ in the following) are currently available in literature, starting from the forties of the last century [18] up to recent results [19]. The experimental data considered in this paper have been selected from a collection of electron-solid interactions experimental databases publicly available $[20,21]$. Considered data also include information on $\eta$ for various materials, focusing in the energy range of the incident electron below about $1 \mathrm{MeV}^{1}$. Moreover an electron

\footnotetext{
${ }^{1}$ This energy limit depends on the particular experiment considered, see Table 1 for more details.
} 

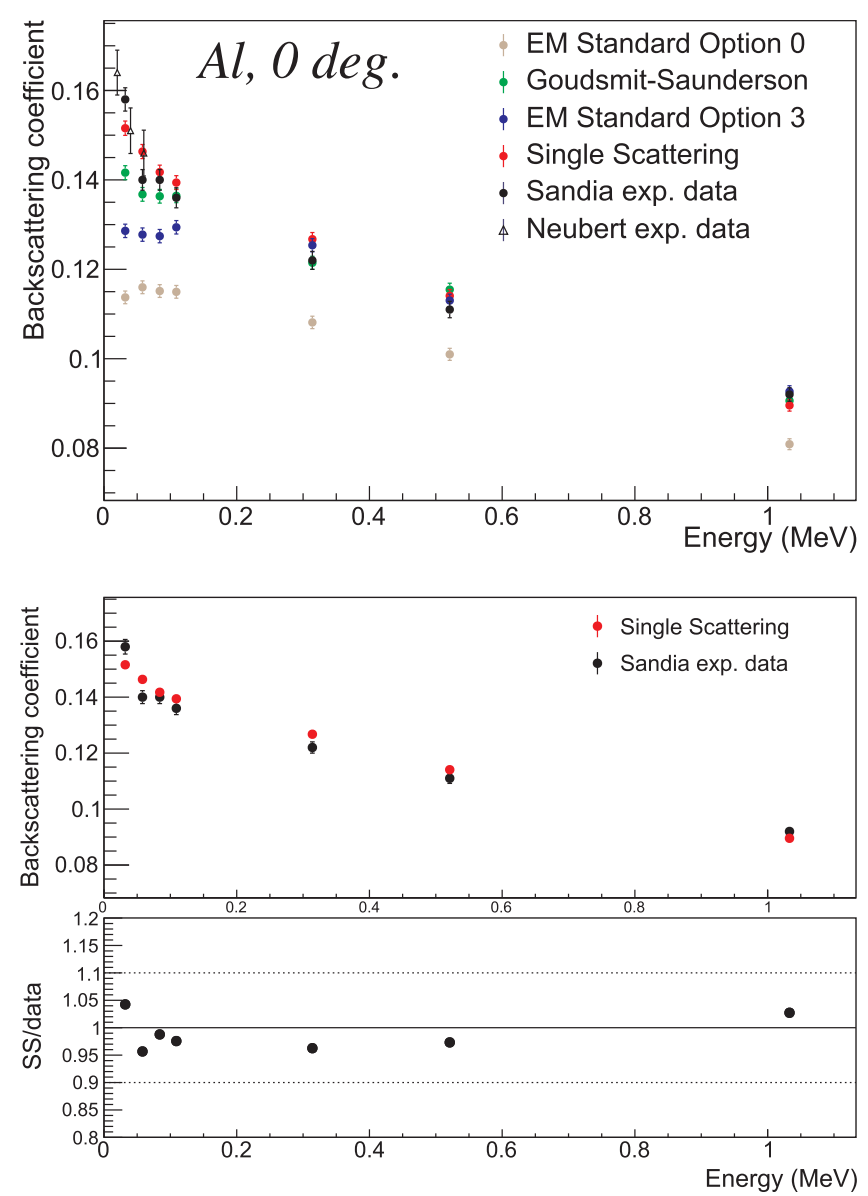

Fig. 4. Backscattering coefficient comparison among Geant4 simulation results for opt0, opt3, GS, and SS physics lists (respectively brown, blue, green, red dots), Sandia [22,23] and Neubert [32] experimental data (black dots and black triangles respectively) for Aluminium at normal incidence angle (upper plot). The ratio between the SS and Sandia data results is shown in the lower plot.

backscattering indirect measurement done by the Sandia Laboratories is considered [22,23], which results are available for various incidence angles and span a wider energy range. A summary of the considered datasets $^{2}$ is reported in Table 1 . In the recent publications $[19,25]$ the backscattering coefficient $\eta$ is conventionally defined as the ratio $I_{B} / I_{P}$, where the current $I_{B}$ measures the number of electrons backscattered from the sample surface with energy greater than $50 \mathrm{eV}$ and the current $I_{P}$ measures the total number of incident electrons. This kind of measurement is usually performed under conventional vacuum conditions $\left(10^{-5}-10^{-7} \mathrm{mbar}\right)$. The main differences among the measured values reported in Table 1 are essentially due to the different experimental techniques and measurement conditions. The principal experimental techniques are briefly described in the following:

1. Polarized grid. A method for the direct evaluation of $\eta$ and the number of secondary electrons $\delta$ uses the device reported in Fig. 3 on the left. A specimen ( $\mathrm{S}$ in the figure) is surrounded by a negatively biased grid $\mathrm{G}(\mathrm{at}-50 \mathrm{~V}$ ) and by a collector $\mathrm{C}$. Because of the grid's electric field the backscattered electrons at the collector will not hit the specimen again. Also the secondary electrons from the specimen will reach the collector without escaping. When the

\footnotetext{
${ }^{2}$ In order to maintain the most possible generality and coherence only two references reported in the cited collection are not considered in this study. The first is an article by Palluel [18], the oldest one in the database, that does not describe the apparatus and the experimental technique used in sufficient detail. The other one is [24], where only two points for $\mathrm{Al}$ are measured and one of them is uncertain.
}
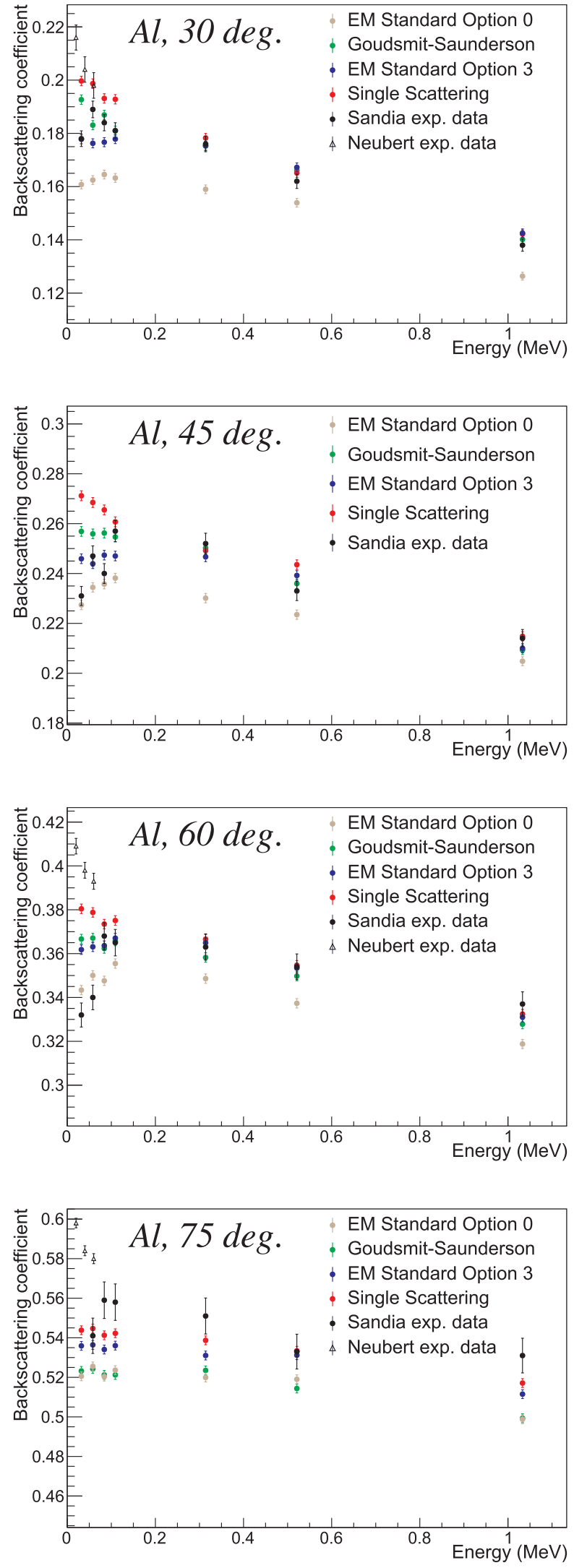

Fig. 5. Comparison among Geant 4 backscattering coefficient simulation for opt0, opt3, GS, and SS physics lists (respectively brown, blue, green, red dots), and Sandia [22,23] and Neubert [32] experimental data (black dots and black triangles respectively) for Aluminium at $30,45,60$, and $75^{\circ}$ incidence angle. 


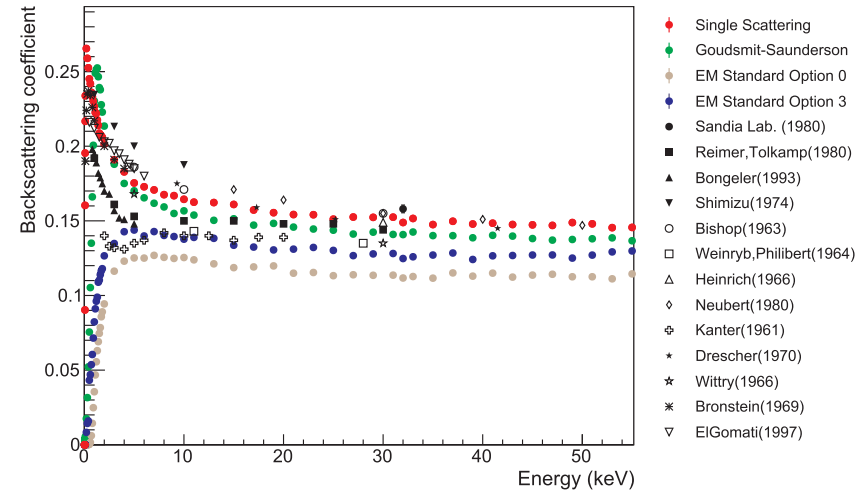

Fig. 6. Geant4 10.3 standard physics lists results are compared with respect to the experimental datasets reported in Table 1 for Aluminium at normal incidence. Note that the default minimum electron energy for the single scattering physics list is $1 \mathrm{keV}$. In this simulation this value was changed to $50 \mathrm{eV}$.

specimen is positively biased a current $I_{+}$is measured at the collector and when the bias is negative $I_{-}$is measured $(50 \mathrm{~V}$ can be neglected compared to the accelerating voltage, of the order of $10 \mathrm{kV}$ ). If $\mathrm{C}, \mathrm{G}$, and $\mathrm{S}$ are electrically connected, the total electron incident probe current $I_{0}$ can be measured. So the backscattering coefficient $\eta$ and the secondary yield $\delta$ can be expressed as $\eta=I_{+} / I_{0}$ and $\delta=\left(I_{-}-I_{+}\right) / I_{0}$. This method is described in detail in $[25,26]$. A recent work adds to this technique an in situ cleaning of the specimen by an energetic ion beam [19]. Note that the results obtained with the polarized grid method need to be scaled proportionally to the grid transparency factor.

2. Polarized single or double target. Another measuring technique, presented in [27], whose experimental setup allows to avoid the corrections for the transparency of the grid, is also considered. In this method the target is positively biased to prevent secondary electrons from leaving the target. As a drawback of this method, the target tends to collect the secondary electrons from the chamber walls as well as secondary electrons from any other obstructions. To solve this issue usually a double target method is used. In this way when the incident electron beam scatters on the first target, the second target is used to measure the background current from the chamber walls (see Fig. 3 on the right).

3. Two regions target. A composite target with two different regions [28] has also been used, with the central area exposed to the electron beam that is electrically isolated from the peripheral area. The currents from the two areas are measured and compared to each other. In particular the one from the outer region is due to the electron backscattering from the chambers walls and other parts of the instrument.

4. Calorimetric measure. The measurement of energy deposition using this method can provide a reliable although indirect estimation of backscattering coefficient $[22,23]$ in case of small bremsstrahlung losses and for material thickness greater than the range of the source electrons.

\subsection{Simulations and data comparison}

\subsubsection{Scattering on Aluminium target}

In Fig. 4 the Geant 4 simulation results for opt0, opt3, GS, and SS physics lists are compared with respect to Sandia data [22,23] for Aluminium $^{3}$ at normal incidence angle (upper plot). An agreement within $10 \%$ is reached for all energies. Above $0.2 \mathrm{MeV}$ the agreement is particularly good, with values below $0.5 \%$. For lower energies (where

\footnotetext{
${ }^{3}$ Aluminium is a relevant material for the ATHENA project and is considered as a benchmark material for the electron backscattering study of this paper.
}
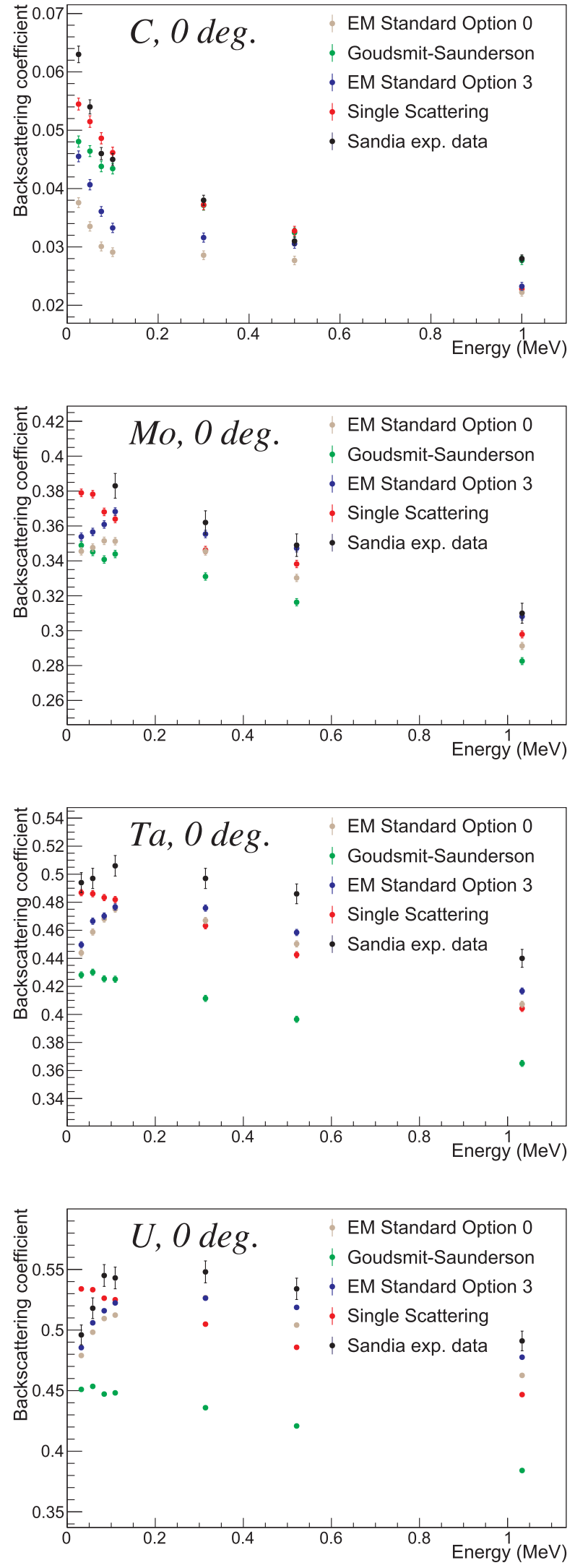

Fig. 7. Carbon (first plot from top), Molybdenum (second plot from top), Tantalum (third plot from top), and Uranium (lower plot) Geant4 simulations for various standard physics lists (colored dots) compared with respect to Sandia Laboratories $[22,23]$ experimental data (black dots). Both simulations and experimental data are at normal incidence angle. 
Silicon, normal incidence angle
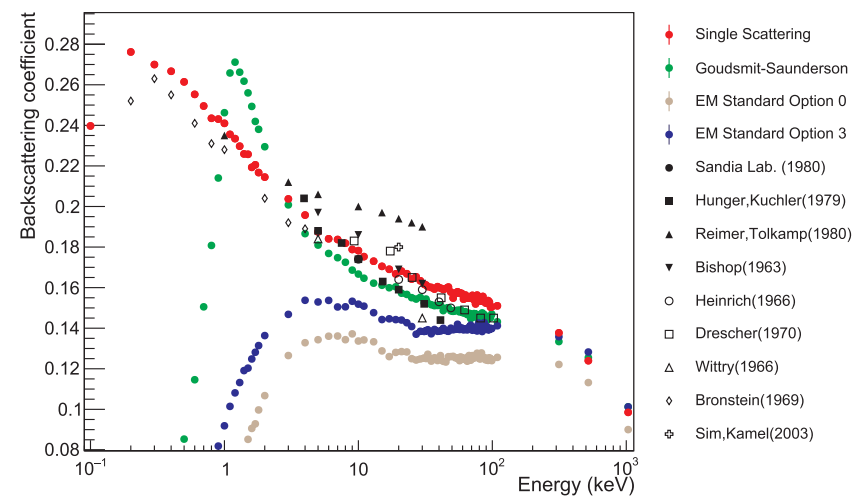

Tungsten, normal incidence angle

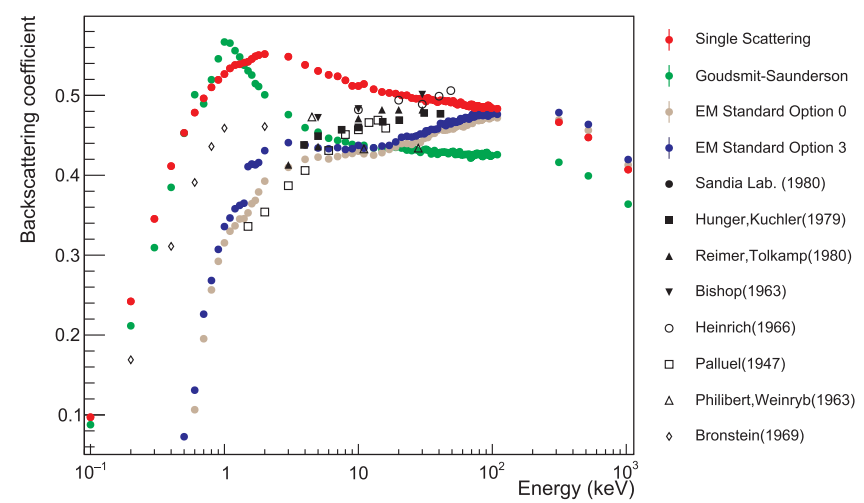

Fig. 8. Geant 4 simulations using different standard electromagnetic physics lists are compared with respect to the experimental datasets reported in Table 1 at normal incidence angle using $\mathrm{Si}$ (upper figure), and Tungsten (lower figure) as a target. The lowest electron energy was set to $50 \mathrm{eV}$.

also Neubert [32] data are shown) SS and GS follow the data peak, whereas opt 0 and opt 3 results present a descending trend. In the lower plot of Fig. 4 the ratio between SS results and Sandia data is shown.

In Fig. 5 the Geant4 simulation results obtained with opt0, opt3, GS, and SS physics lists are compared with respect to Sandia [22,23] and Neubert [32] experimental data. Scattering on Aluminium at 30, 45, 60, $75^{\circ}$ of incidence angle is considered. As the angle increases, Sandia and Neubert results show a different behavior. Sandia data have a descending shape below $0.2 \mathrm{MeV}$ whereas the Neubert ones remain high with a shape similar to the $0^{\circ}$ case. The Sandia shape at low energies disagrees also with respect to the theoretical prediction of the Monte Carlo simulation used in the original article [23]. This discrepancy is addressed in the conclusions of the article itself, where some possible systematic sources are discussed. Summarizing, from all these comparisons seems that the single scattering process gives the better description of experimental data, followed by GS. The latter physics list is noticeably faster than SS (a factor about 20) in the execution of the simulation.

To investigate deeply the reliability of the backscattering simulation in the low energy range a comparison among the Geant4 single scattering results and the experimental datasets reported in the C. Joy collection has been performed. A case study of Aluminium at normal incidence is considered. In Fig. 6 the simulation results obtained using opt0, opt3, GS, and SS physics lists are compared with respect to the experimental datasets reported in Table 1 . All the experimental data present a threshold on the electron energy, varying between about 50 and $100 \mathrm{eV}$ depending on the particular setup considered. A cut on the
Gold, normal incidence angle

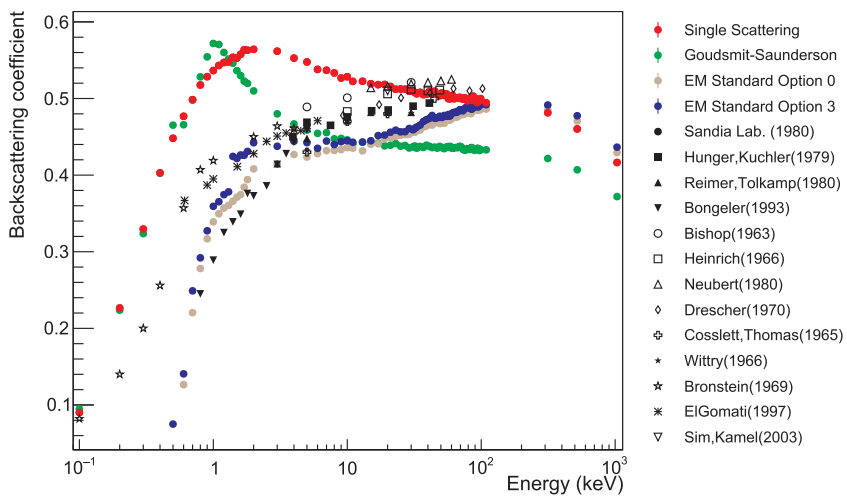

Copper, normal incidence angle

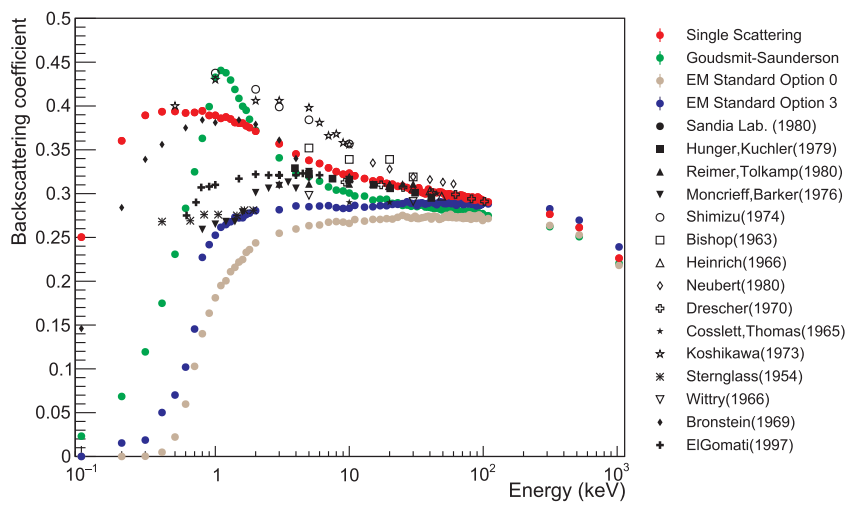

Fig. 9. Geant4 simulations using different standard electromagnetic physics lists are compared with respect to the experimental datasets reported in Table 1 at normal incidence angle using Au (upper figure), and Cu (lower figure) as a target. The lowest electron energy was set to $50 \mathrm{eV}$.

minimum electron energy was used in the Geant4 simulations to reproduce that experimental condition. A minimum value equal to $50 \mathrm{eV}$ was used in the plot of Fig. 6, where SS and GS simulations are in the middle of the experimental data dispersion. SS can reproduce slightly better the peak at low energy, whereas opt 0 and opt 3 underestimate the backscattering fraction below about $5-10 \mathrm{keV}$. Several factors can influence the backscattering measurement, e.g. the data taking technique, the cleaning of the sample, the correct estimation of systematic sources. Despite of the range of variation of the experimental results (up to 60\% difference in the lower energy region), the SS agrees very well with the monotonic increasing shape below $0.02 \mathrm{MeV}$ and the horizontally asymptotic trend for energy above $0.03 \mathrm{MeV}$.

\subsubsection{Scattering dependence on target material}

A further study has been performed comparing the simulation with respect to experimental data from Sandia Laboratories [22,23] for scattering on various target materials. In Fig. 7 the results for Carbon (upper left), Molybdenum (upper right), Tantalum (lower left), and Uranium (lower right) are shown for normal incidence angle. The experimental distribution at low energy (below about $150 \mathrm{keV}$ ) seems to be dependent on the atomic number. For low atomic numbers ( $\mathrm{Al}$ and C) the trend is increasing, whereas for $\mathrm{U}$ and Ta it is decreasing.

\subsubsection{Further studies on low energy trend}

From the results reported above, the low energy region below $100 \mathrm{keV}$ seems to be particularly sensitive to the experimental features of each dataset considered. In order to obtain the best possible 
Single Scattering, different minimum electron energy

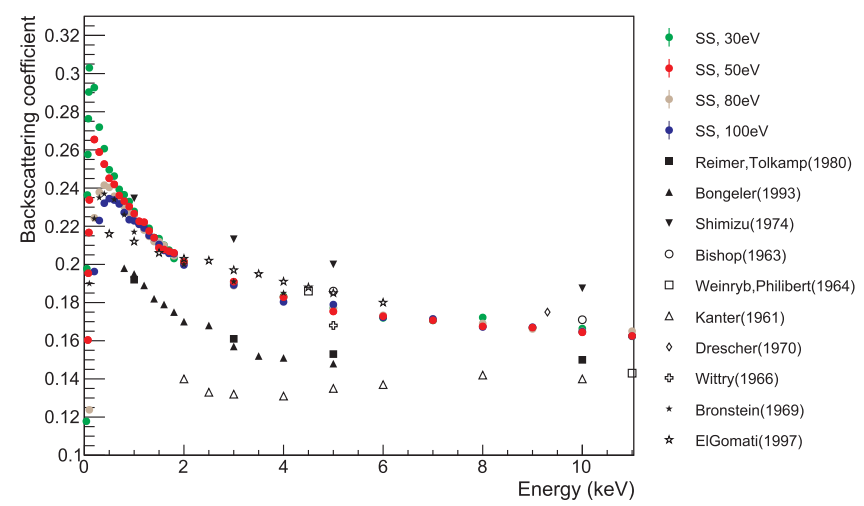

Fig. 10. The Geant4 10.3 Single Scattering physics list results are compared with respect to the experimental datasets reported in Table 1 for Aluminium at normal incidence, varying the minimum electron energy threshold. The minimum electron energy values used in the simulation are $30 \mathrm{eV}$ (green dots), $50 \mathrm{eV}$ (red dots), $80 \mathrm{eV}$ (grey dots), and $100 \mathrm{eV}$ (blue dots). (For interpretation of the references to colour in this figure legend, the reader is referred to the web version of this article.)

agreement with respect to each particular dataset, dedicated simulations should be carried on, addressing separately the various cases. Thus in the present study it is possible to perform only a qualitative comparison between the various experimental datasets and the Geant4 simulation results, in order to address the reliability and stability of the Geant4 standard physics in this extremely low energy regime. For this purpose a set of simulations at low energy for various materials has been carried on. Backscattering from $\mathrm{Si}$ and $\mathrm{W}$ is reported in Fig. 8 (upper and lower plot respectively), whereas from $\mathrm{Au}$ and $\mathrm{Cu}$ in Fig. 9 (upper and lower plot respectively). The agreement is in general good, with SS and GS staying in the middle of the experimental data spectrum. Opt0 and opt 3 seem to underestimate the number of backscattered electrons below about $10 \mathrm{keV}$. All the tested physics lists can reproduce well the change of shape around $1 \mathrm{keV}$ for $\mathrm{W}, \mathrm{Au}$, and $\mathrm{Cu}$. The Si sample presents an ascending trend down to about $0.5 \mathrm{keV}$, optimally described by SS.

The behaviour of the Geant4 standard physics lists at these extremely low energies, and consequently the agreement with respect to the experimental data, is influenced by the minimum electron energy cut used in the simulation. As discussed above, all the experiments considered in this study use an effective energy threshold for electrons, that can varies from about $50 \mathrm{eV}$ to about $1 \mathrm{keV}$ depending on the specific configuration. The effects of the variation of this cut in the simulations are shown in Fig. 10 for Single Scattering. The SS low energy tail changes considerably together with the lowest electron energy value. A cut between 50 and $80 \mathrm{eV}$ can assures an accurate agreement with respect to the considered experimental data, in harmony with the value effectively used in the experiments. It is important to note that the default value in the standard SS physics list is $1 \mathrm{keV}$. The tests performed in this study thus evaluate the single scattering in a frontier region, obtaining excellent results and confirming the reliability of this model for such conditions.

\section{Conclusions}

In this paper the backscattering of electron has been analysed in detail and compared with respect to the Geant4 10.3 simulation results. SS physics list reproduces very well the experimental data in both the relatively high (from $0.1 \mathrm{MeV}$ to $1 \mathrm{MeV}$ ) and low energy region (from $0.1 \mathrm{keV}$ to $100 \mathrm{keV}$ ). The agreement is excellent for low atomic number materials like Aluminium and Carbon and for normal incidence data. Also the GS physics list shows a good agreement and presents the advantage to be less time consuming from the computational point of view. Below $100 \mathrm{keV}$ discrepancies among simulations and part of the experimental datasets are shown, but it should be considered that in this region the data taking conditions and the experimental features strongly influence the results. Thus, it is not possible to make a direct comparison without knowing the experimental setups in details and performing a dedicated simulation for each individual case. In conclusion, in this study a very good agreement has been found with respect to several experimental results, figuring the Geant4 electron backscattering simulation to be robust and reliable above about $100 \mathrm{keV}$. Despite that below this energy the backscattering coefficient is strongly influenced by the experimental conditions, a general acceptable agreement has been found in the range from $0.1 \mathrm{keV}$ to $100 \mathrm{keV}$. Collectively those results cover the electrons energy regime that gives an important contribution to the whole ATHENA background and state the capability to reproduce the electron scattering processes happening on the X-ray instruments shielding with a very good precision.

\section{Acknowledgements}

The research leading to these results has received funding from ESA (European Space Agency) under the AREMBES consortium (ESA Contract No. 4000116655/16/NL/BW) within the ATHENA X-ray Observatory project.

\section{References}

[1] K. Nandra, et al., The hot and energetic universe: a white paper presenting the science theme motivating the Athena + mission arXiv:1306.2307.

[2] S. Agostinelli, et al., Geanta simulation toolkit, Nucl. Instr. Meth. Phys. Res. Sect. A 506 (3) (2003) 250-303, http://dx.doi.org/10.1016/S0168-9002(03)01368-8.

[3] J. Allison, et al., Geant4 developments and applications, IEEE Trans. Nucl. Sci. 53 (1) (2006) 270-278, http://dx.doi.org/10.1109/TNS.2006.869826.

[4] J. Allison, et al., Recent developments in geant4, Nucl. Instr. Meth. Phys. Res. Sect. A 835 (2016) 186-225, http://dx.doi.org/10.1016/j.nima.2016.06.125.

[5] V.N. Ivanchenko, O. Kadri, M. Maire, L. Urban, Geant4 models for simulation of multiple scattering, J. Phys.: Conf. Ser. 219 (3) (2010) 032045 URL:http://stacks. iop.org $/ 1742-6596 / 219 / \mathrm{i}=3 / \mathrm{a}=032045$.

[6] S. Lotti, E. Perinati, L. Natalucci, L. Piro, T. Mineo, L. Colasanti, C. Macculi, Estimate of the impact of background particles on the x-ray microcalorimeter spectrometer on ixo, Nucl. Instr. Meth. Phys. Res. Sect. A 686 (Suppl. C) (2012) 31-37, http://dx.doi.org/10.1016/j.nima.2012.05.055 URL: < http://www. sciencedirect.com/science/article/pii/S0168900212005554 >

[7] N. Meidinger, J. Eder, T. Eraerds, K. Nandra, D. Pietschner, M. Plattner, A. Rau, R. Strecker, The Wide Field Imager Instrument for Athena, ArXiv e-prints arXiv:1702. 01079.

[8] L. Urbn, A model for multiple scattering in GEANT4, Tech. Rep. CERN-OPEN-2006077, CERN, Geneva (Dec 2006). URL: < http://cds.cern.ch/record/1004190 >

[9] H.W. Lewis, Multiple scattering in an infinite medium, Phys. Rev. 78 (1950) 526-529.

[10] O. Kadri, V. Ivanchenko, F. Gharbi, A. Trabelsi, Incorporation of the goudsmit saunderson electron transport theory in the geant4 monte carlo code, Nucl. Instr. Meth. Phys. Res. Sect. B 267 (23-24) (2009) 3624-3632, http://dx.doi.org/10. 1016/j.nimb.2009.09.015 URL: < http://www.sciencedirect.com/science/article/ pii/S0168583X09009823 > .

[11] I. Kawrakow, A.F. Bielajew, On the representation of electron multiple elasticscattering distributions for monte carlo calculations, Nucl. Instr. Meth. Phys. Res. Sect. B 134 (3-4) (1998) 325-336, http://dx.doi.org/10.1016/S0168-583X(97) 00723-4.

[12] A. Bagulya, et al., Recent progress of geant4 electromagnetic physics for lhe and other applications, accepted for Journal of Physics: Conference Series, CHEP-2016.

[13] M.J. Boschini, C. Consoland, M. Gervasi, S. Giani, D. Grandi, V. Ivanchenko, P. Nieminem, S. Pensotti, P.G. Rancoita, M. Tacconi, An expression for the Mott cross section of electrons and positrons on nuclei with $\mathrm{Z}$ up t0 118, Rad. Phys. Chem. 90 (2013) 39-66, http://dx.doi.org/10.1016/j.radphyschem.2013.04.020 arXiv:1304.5871.

[14] C.K. Ross, M.R. McEwen, A.F. McDonald, C.D. Cojocaru, B.A. Faddegon, Measurement of multiple scattering of 13 and $20 \mathrm{mev}$ electrons by thin foils, Med. Phys. 35 (9) (2008) 4121-4131.

[15] E.M. Lyman, A.O. Hanson, M.B. Scott, Scattering of 15.7-mev electrons by nuclei, Phys. Rev. 84 (1951) 626-634, http://dx.doi.org/10.1103/PhysRev. 84.626.

[16] Geant4 physic validation and verification page. URL: < http://geant4.web.cern.ch/ geant4/results/validationplots/thintarget/hadronic/mediumenergy/test1/ 113800MeVp.shtml > .

[17] G.J. Lockwood, et al., Calorimetric measurement of electron energy deposition in extended media, SANDIA REPORT SAND79-0414.UC-34a.

[18] P. Palluel, Composante rediffusee du rayonnement electronique secondaire des 
metaux, Comp. Rendus de l'Acad. Sci. 224 (1947) 1492.

[19] M. El Gomati, A. Assad, Backscattering coefficients for low energy electrons, in: Proc. 5th European Workshop on Microbeam Analysis.

[20] D.C. Joy, A database on electron-solid interactions, Scanning 17 (5) (1995) 270-275, http://dx.doi.org/10.1002/sca.4950170501.

[21] D.C. Joy, A database of electron-solid interactions, 2008. web.utk.edu/ srcutk/database.doc.

[22] G.J. Lockwood, et al., Calorimetric Measurement of Electron Energy Deposition in Extended Media Theory vs Experiment, SAND79-Report, Sandia National Laboratories, Albuquerque.

[23] G.J. Lockwood, et al., Electron Energy and Charge Albedos Calorimetric Measurement vs Monte Carlo Theory, SAND80-Report, Sandia National Laboratories, Albuquerque.

[24] V.E. Cosslett, R.N. Thomas, Multiple scattering of 5-30 kev electrons in evaporated metal films iii: Backscattering and absorption, Brit. J. Appl. Phys. 16 (6) (1965) 779 URL: < http://stacks.iop.org/0508-3443/16/i=6/a=303 >

[25] L. Reimer, C. Tollkamp, Measuring the backscattering coefficient and secondary electron yield inside a scanning electron microscope, Scanning 3 (1) (1980) 35-39, http://dx.doi.org/10.1002/sca.4950030105.

[26] R. Bngeler, U. Golla, M. Kssens, L. Reimer, B. Schindler, R. Senkel, M. Spranck, Electron-specimen interactions in low-voltage scanning electron microscopy, Scanning 15 (1) (1993) 1-18, http://dx.doi.org/10.1002/sca.4950150102.

[27] H.E. Bishop, Electron scattering and X-ray production (Ph.D. thesis), 1966

[28] K.F.J. Heinrich, Electron probe microanalysis by specimen current measurement, in: Proc. 4th Conf. on X-ray Optics and Microanalysis.

[29] R. Shimizu, Secondary electron yield with primary electron beam of kilo electron volts, J. Appl. Phys. 45 (5) (1974) 2107-2111, http://dx.doi.org/10.1063/1. 1663552.

[30] T. Koshikawa, R. Shimizu, Secondary electron and backscattering measurements for polycrystalline copper with a spherical retarding-field analyser, J. Phys. D: Appl. Phys. 6 (11) (1973) 1369 URL: < http://stacks.iop.org/0022-3727/6/i=11/a = $312>$.

[31] J.P.E. Weinryb, Mesure du coefficient de retrodiffusion des electrons de 5 a $30 \mathrm{kev}$, Compt. Rendus Acad. Sci. Paris 258 (1964) 4535-4538.

[32] G. Neubert, S. Rogaschewski, Backscattering coefficient measurements of 15 to 60 kev electrons for solids at various angles of incidence, Phys. Status Solidi (a) 59 (1) (1980) 35-41.

[33] H. Kanter, Contribution of backscattered electrons to secondary electron formation, Phys. Rev. 121 (1961) 681-684, http://dx.doi.org/10.1103/PhysRev.121.681.

[34] H. Kanter, Energy dissipation and secondary electron emission in solids, Phys. Rev. 121 (1961) 677-681, http://dx.doi.org/10.1103/PhysRev.121.677.

[35] H. Drescher, L. Reimer, H. Seidel, Backscattering and secondary electron emission of 10-100 kev electrons and correlations to scanning electron microscopy, Z. Angew. Phys. 29 (1970) 331-336.

[36] D.B. Wittry, Secondary electron emission in the electron probe, in: Proc. 4th Conf. on X-ray Optics and Microanalysis, 1966, pp. 168-180.

[37] I.M. Bronstein, B.S. Fraiman, Vtorichnaya elektronnaya emissiya (secondary electron emission), Nauka, Moskva, 1969, p. 340. 